\title{
适用于月面极端地形的爬-滚机器人设计及爬 行滚动特性分析
}

\author{
张元勋 ${ }^{1,2}$ 黄泽东 ${ }^{2,3}$ 韩亮亮 ${ }^{4,5}$ 谷程鹏 ${ }^{4,5}$ 张文奇 ${ }^{4,5}$ \\ (1. 重庆大学航空航天学院 重庆 400044; \\ 2. 重庆大学教育部深空探测联合研究中心 重庆 400044; \\ 3. 重庆大学机械工程学院 重庆 400044; \\ 4. 上海宇航系统工程研究所 上海 201108;
}

5. 中国航天科技集团有限公司空间结构与机构技术实验室 上海 201109)

\begin{abstract}
摘要: 面向月面极端环境科学探测任务, 提出一种兼有快速通过性和良好环境适应性的爬行滚动一体化机器人设计方案, 通过 机器人 6 条腿的形态变化, 使机器人具有爬行和滚动两种运动模式。在对爬-滚机器人构型分析的基础上, 建立了单腿正逆运动 学模型, 以运动学为基础, 基于足端轨迹规划设计了爬行模式下机器人直行、原地转向步态; 结合非结构地形的特殊性, 通过 尺寸和力约束条件分析了机器人滚动模式下的最大爬坡角和最大越障高度。基于 ADAMS 软件对机器人爬行模式直行、原地转 向, 滚动模式爬坡、越障等运动行为进行了模拟, 并搭建了机器人移动试验平台, 对机器人样机在实验室环境中进行了测试, 仿真和试验结果表明机器人在爬行模式下具有良好的移动性能, 在滚动模式下具有一定的爬坡和越障能力, 爬行、滚动模式运 动特性分析能准确地评价机器人性能，证明了该设计方案的适用性和可行性，为后续特种探测机器人的研究提供借鉴。
\end{abstract}

关键词: 月面; 机器人; 构型设计; 异型轮; 运动特性

中国分类号: TG156

\section{Design and Analysis of the Crawling and Rolling Characteristics of the Crawling and Rolling Robot for the Lunar Extreme Terrain}

\author{
ZHANG Yuanxun $^{1,2}$ HUANG Zedong ${ }^{2,3}$ HAN Liangliang ${ }^{4,5}$ GU Chengpeng ${ }^{4,5}$ \\ ZHANG Wenqi ${ }^{4,5}$
}

(1. College of Aerospace Engineering, Chongqing University, Chongqing 400044;

2. Center of Space Exploration of Ministry of Education, Chongqing University, Chongqing 400044;

3. College of Mechanical Engineering, Chongqing University, Chongqing 400044;

4. Aerospace System Engineering Shanghai, Shanghai 201108;

5. Space Structure and Mechanism Technology Laboratory of China Aerospace Science and Technology Group Co.

Ltd., Shanghai 201109)

\begin{abstract}
For the scientific exploration task of lunar extreme environment, a scheme of the crawling and rolling integrated robot with fast passing and good environmental adaptability is proposed. Through the shape change of six legs of the robot, the robot has two motion modes of crawling and rolling. Based on the analysis of the configuration of the climbing roll robot, the forward and inverse kinematics model of the single leg is established. Based on the kinematics and the foot end trajectory planning, the straight and in-situ turning gait of the robot in the crawling mode is designed. Combined with the particularity of the complex terrain, the maximum
\end{abstract}

* 国家自然科学基金(51705045)、民用航天技术预告研究(D030103) 和载人航天预告研究(030601)资助项目。20200324 收到初稿, 20200720 收到修改稿 
climbing angle and the maximum obstacle crossing height of the robot in the rolling mode are analyzed by size and force constraints. Based on the ADAMS software, the movement behaviors of robot, such as straight gait and turning gait in the crawling mode, climbing and obstacle crossing in the rolling mode, are simulated, and the robot prototype test platform in the laboratory environment is built. The simulation and test results show that the robot has good mobile performance in the crawling mode, and has certain climbing performance and obstacle surmounting ability in the rolling mode, crawling and rolling mode motion characteristics analysis can accurately evaluate the performance of the robot, which proves the applicability and feasibility of the design scheme, and provides reference for the follow-up research of special detection robot.

Key words: lunar surface; robot; configuration design; special-shaped wheel; motion characteristics

\section{0 前言}

月球探测机器人作为探月研究的重要载体工具, 对于人类探索月球环境、开展科学研究具有重要意 义。月球复杂恶劣的表面环境对月表探测机器人的 构型、可靠性、环境适应性等有着较高的要求。针 对常规星表地形, 前苏联的月球车(Lunokhod 1、 Lunokhod 2), 美国的阿波罗载人月球车(Apollo 15 17), 中国的玉兔月球车 (图 $1 \mathrm{a}$ 所示为玉兔二号) 等为代表的轮式月面探测机器人已经成功着陆月球 并开展了较为广泛的科学探测工作。其中, 玉兔月 球车的移动系统为六轮摇臂式机构, 属于典型的轮 式移动机器人, 在星表常规地形下具有较高的可靠 性和稳定性 ${ }^{[1]}$ 。

对于星表极端地形，如斜坡、障碍、松软路面、 沟壑、凹凸起伏大等地形 ${ }^{[2]}$, 目前的轮式构型机器 人难以承担此类环境下的科学探测任务, 因此, 开 展运动灵活、环境适应能力强的新型特种机器人的 研究尤为迫切。目前针对非结构地形, 常用的移动 构型方式有履带式、腿式、轮腿式等。履带式移动 机器人典型代表有美国的 Packbot 履带式机器人(图 1a $)^{[3]}$, 该机器人有六条履带, 支撑面积大, 下陷度 小, 运动非常平稳, 适用地形范围较广; 其不足之 处为传动效率低, 运动不够灵活, 转弯半径大。腿 式机器人的代表有波士顿动力的 Spotmini(图 1b) ${ }^{[4]}$, ESA 的 SpaceBok(图 1c) ${ }^{[5]}$ 等, 腿式机器人具备全地 形适应能力, 在非结构地形环境下展现出更优越的 通过性能。然而, 腿式机器人因其控制和结构复杂, 往往越障效率和速度较低。

综合轮式和腿式机器人构型的优缺点, 将轮 式和腿式构型相结合是星表机器人构型研究的方 向之一。美国 NASA 研制的 ATHLETE ${ }^{[6]}$ 、德国 Festo 公司设计的 BionicWheelBot(图 1d) ${ }^{[7]}$ 都是将 轮式和腿式构型组合成的典型轮腿式机器人, 其 中 BionicWheelBot 机器人采用多连杆和线绳耦合
而成的辅助腿结构为机器人的轮式移动提供助力。 国内丁希仑等 ${ }^{[9]}$ 先后提出了多种轮腿式机器人方 案, 并对其运动学及运动步态进行了研究。轮腿 式构型具有较好的移动性、通过性和环境适应性, 但目前大多数轮腿式机器人构型方案采用在腿式 机器人的腿部添加一个驱动轮, 轮和腿结构和功 能互相分离的策略 ${ }^{[10-11]}$, 增加了驱动电动机数量, 控制难度较大。

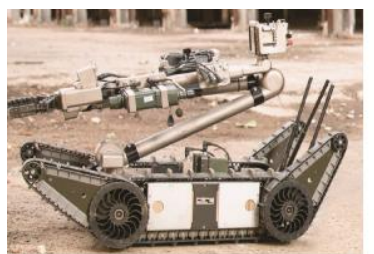

(a) Packbot 履带车

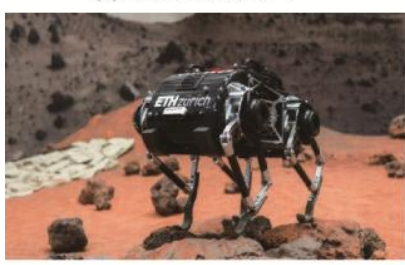

(c) SpaceBok

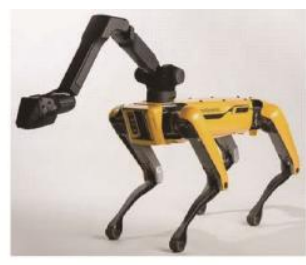

(b) Spotmini

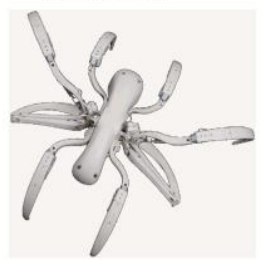

(d) BionicWheelBot
图 1 多种构型形式机器人

在上述分析的基础上, 本文提出了一种新型的 轮腿机器人设计方案——爬-滚机器人, 该机器人既 具有类似腿式机器人的爬行模式, 也可以通过腿的 形态变化构成 “轮” , 实现滚动模式。通过理论分 析、仿真与试验相结合的方式分析评估了爬-滚机器 人爬行模式的移动性能和滚动模式的运动性能, 验 证了设计方案的可行性和正确性。

\section{1 爬-滚机器人整体方案设计}

爬-滚机器人结构设计方案和原理如图 2 所示, 包括前腿、中腿、后腿、躯体、控制器、辅助腿组 件、电池等零部件。机器人躯体平台为长方体结构。 前腿、后腿为两自由度的关节连杆串联结构, 中腿 为三自由度关节连杆串联结构, 六条腿对称布置于 
躯体平台两侧, 序号分别为 $1,2, \cdots, 6$ 。图 3 所示 为辅助腿空间多连杆复合机构, 布置在躯体平台下 方, 由曲柄摇杆机构、空间 4R1S 机构、双摇杆机 构耦合而成, 电动机安装在曲柄摇杆机构的曲柄处, 通过 4R1S 空间机构传递力和力矩给两个双摇杆 机构。

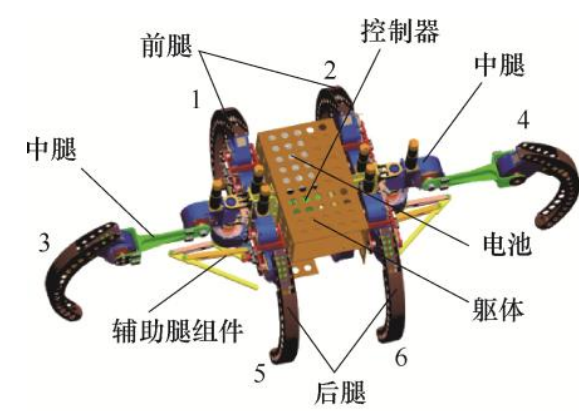

(a) 爬-滚机器人设计方案

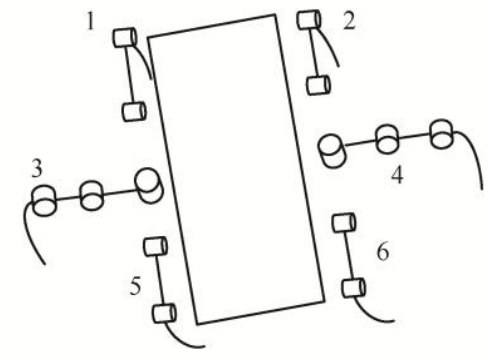

(b) 爬-滚机器人结构简图

图 2 爬-滚机器人设计方案

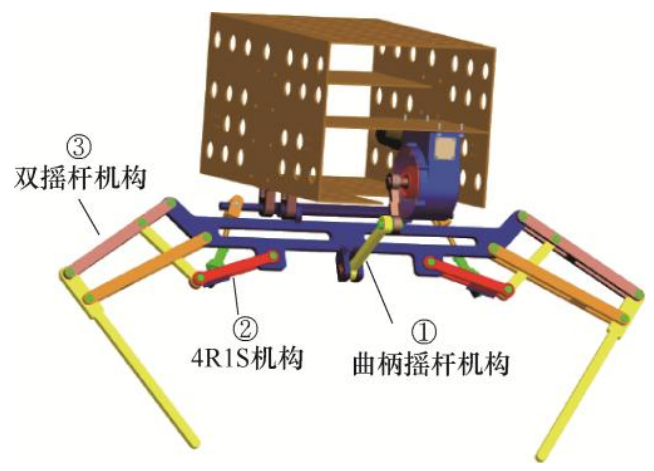

图 3 机器人辅助腿组件

在爬-滚机器人整体设计方案中, 腿组件主要 有关节驱动模块、连杆、圆弧形腿足等零部件。 关节驱动模块有伺服电动机、蜗轮蜗杆传动件, 具有较好的承载能力和自锁性能。机器人六条腿 对称交替布置, 腿足采用圆弧形结构。电源、控 制器、传感器集中布置于躯体围成的腔体内, 便 于防护设计。整个机器人共 15 个自由度, 由 15 个伺服电动机独立控制, 相比目前已有的爬行类、 轮腿式机器人, 驱动自由度较少, 控制简单, 结 构紧凑。
爬-滚机器人能够在爬行模式和滚动模式之间 切换, 同时具备轮式机器人的快速移动能力和腿式 机器人的地形适应能力。图 2 所示的机器人状态为 基础运动模式, 即爬行模式。在多数环境中, 机器 人采用基础运动模式, 利用 6 个爬行腿交替运动完 成爬行, 可以获得较好的稳定性和地形适应能力。 在需要执行快速移动的任务时, 如平坦路面或下坡 路段, 通过调整机器人腿的形态, 可切换成图 4 的 滚动模式, 在辅助腿的推动下向前滚动, 实现快速 移动。

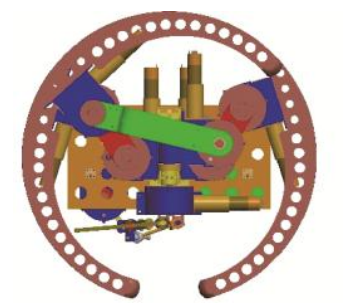

(a) 机器人滚动形态, 从侧 面看去呈 “轮”形

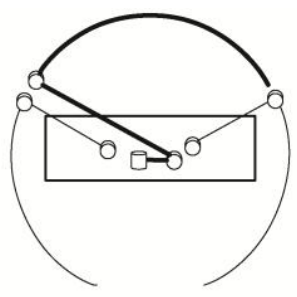

(b) 加粗的线代表中腿, 与 前后腿在空间上错开
图 4 机器人滚动形态

图 5 演示了机器人在平地和下坡路段采取滚动 模式, 在上坡路段采取爬行模式, 实现兼顾机器人 移动效率和环境适应特性。

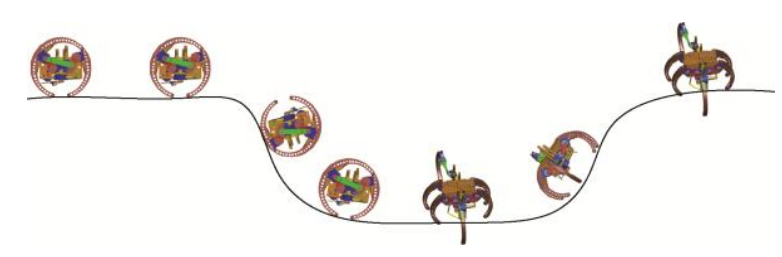

图 5 机器人在不同环境中的运动模式

机器人处于滚动模式时, 如图 6 所示, 图 $6 \mathrm{a}$ 表示机器人辅助腿就位, 此时辅助腿的末端连杆刚 触地; 图 $6 \mathrm{~b}$ 表示辅助腿工作状态, 此时辅助腿电动 机继续施加力矩, 辅助腿的末端连杆施加机器人反 向作用力, 不断提高机器人的速度, 当辅助腿上双 摇杆机构伸展到极限时, 辅助腿离开地面, 机器人 在惯性作用下向前滚动; 图 $6 \mathrm{c}$ 表示辅助腿离地时的 状态, 此时辅助腿电动机继续施加力矩, 辅助腿的 连杆逐渐收回; 图 6d 表示机器人收回辅助腿的状态, 在滚动结束后辅助腿回到初始位置, 准备下一次推 动机器人滚动。辅助腿通过上述运动, 实现曲柄摇 杆机构上布置的电动机仅沿一个旋转方向转动, 而 双摇杆机构能往复拨动, 具有较大的伸缩比和推动 力。 


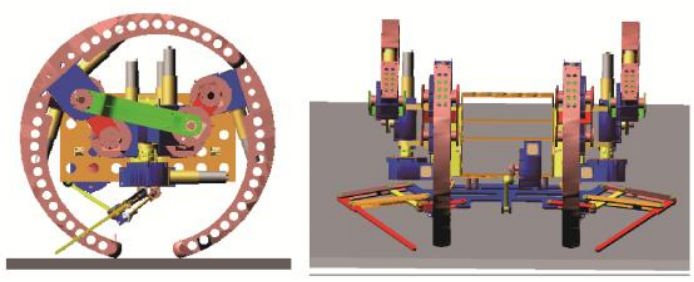

(a) 辅助腿就位

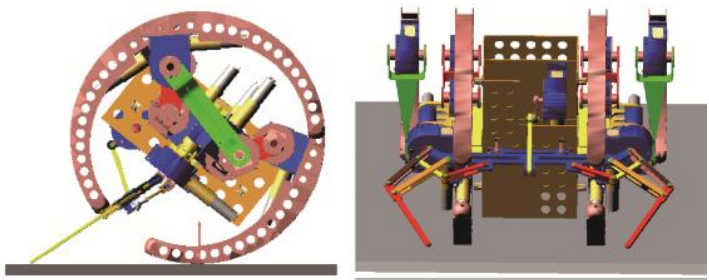

(b) 辅助腿工作

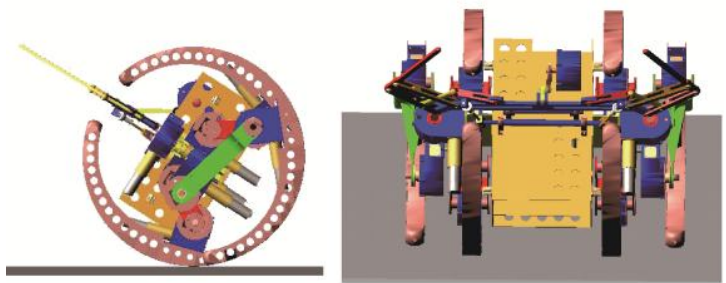

(c) 辅助腿离地

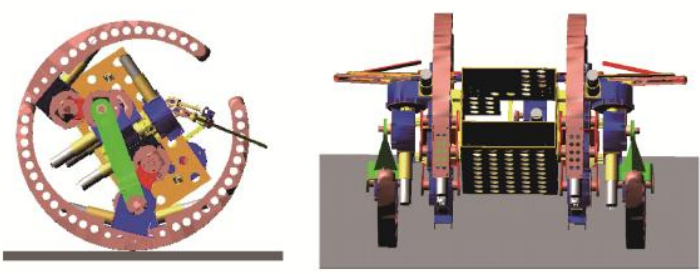

(d) 辅助腿收回

图 6 辅助腿推动机器人滚动过程

机器人部分设计参数见表 1 。

表 1 机器人部分结构参数

\begin{tabular}{cc}
\hline 参数 & 数值 \\
\hline 躯体 $/\left(\frac{\text { 长 }}{\mathrm{mm}} \times \frac{\text { 宽 }}{\mathrm{mm}} \times \frac{\text { 高 }}{\mathrm{mm}}\right)$ & $310 \times 180 \times 160$ \\
滚动模式轮直径 $/ \mathrm{mm}$ & 450 \\
躯体、腿、各杆材料 & 铝合金 \\
\hline
\end{tabular}

\section{2 机器人的运动分析}

爬-滚机器人具有爬行和滚动两种运动模式, 明 确运动参数与机器人关节角和输出力矩之间的关系 规律, 对于不同工作环境和任务需求采用的控制策 略具有重要的意义。

\section{1 爬行模式运动分析}

爬行模式是机器人基础运动模式, 为分析机器 人爬行过程中各足端点与机器人躯体的位置关系, 建立如图 7 所示机器人坐标系, 定义大地坐标系为
$\left\{\Sigma_{\mathrm{w}}\right\}, x_{\mathrm{w}}$ 指向机器人初始时刻水平前进方向, $z_{\mathrm{w}}$ 沿重力反方向, $y_{\mathrm{w}}$ 由右手定则确定。爬-滚机器人 躯体可以视为一个长方体, 因机器人对称布局, 质 量主要集中在躯体, 因此可视机器人质心与躯体形 心重合，定义过机体形心的躯体坐标系 $\left\{\Sigma_{\mathrm{B}}\right\}$ ，其坐 标原点 $O_{\mathrm{B}}$ 与机器人的躯体几何中心重合, $z_{\mathrm{B}}$ 轴垂 直于躯体上表面向上， $x_{\mathrm{B}}$ 垂直于机器人前表面指向 机器人前进方向, $y_{\mathrm{B}}$ 由右手定则确定。建立各腿关 节 D-H 坐标系 $\left\{\Sigma_{i 0}\right\} 、\left\{\Sigma_{i 1}\right\}(i=1,2, \cdots, 6)$, 以及中腿 坐标系 $\left\{\Sigma_{i 2}\right\} \quad(i=3,4), i$ 表示机器人腿序号。

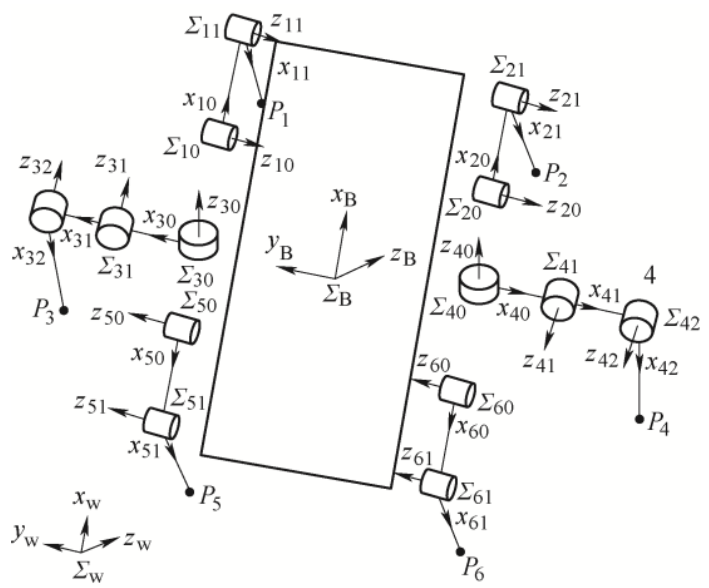

图 7 机器人整体坐标系

各腿坐标系 $\left\{\Sigma_{i 0}\right\}(i=1,2, \cdots, 6)$ 可以看成躯体坐 标系 $\Sigma_{\mathrm{B}}$ 先沿自身坐标系平移一个 $\left(x_{i} y_{i} z_{i}\right)$ $(i=1,2, \cdots, 6)$ 坐标值后, 再绕自身坐标系的 $z$ 轴旋转 $\alpha_{i}, x$ 轴旋转 $\beta_{i}$ 后得到。身区体坐标系 $\left\{\Sigma_{\mathrm{B}}\right\}$ 到各腿坐 标系 $\left\{\Sigma_{i 0}\right\}(i=1,2, \cdots, 6)$ 的变换矩阵 ${ }^{[12]}$ 为

$$
{ }_{i 0}^{B} \boldsymbol{T}=\operatorname{Trans}\left(x_{i}, y_{i}, z_{i}\right) \operatorname{Rot} z\left(\alpha_{i}\right) \operatorname{Rot} x\left(\beta_{i}\right)
$$

从而建立了躯体坐标系 $\left\{\Sigma_{\mathrm{B}}\right\}$ 与各腿坐标系 $\left\{\Sigma_{i 0}\right\}(i=1,2, \cdots$, , 的之间的关系。

\subsection{1 前后腿运动学}

在分析前后腿的位置关系时, 以图 2 所示的 2 号腿为例, 可以将其视为 2 自由度串联机械臂, 如 图 8 所示。腿两个连杆的长度分别为 $a_{1} 、 a_{2}$, 各个 驱动关节的角度为 $\theta_{1} 、 \theta_{2}$, 关节角 $\theta_{1} 、 \theta_{2}$ 符号根据 右手定则确定，即以右手拇指指向关节轴线的正方 向，四指弯曲的方向为关节正方向。
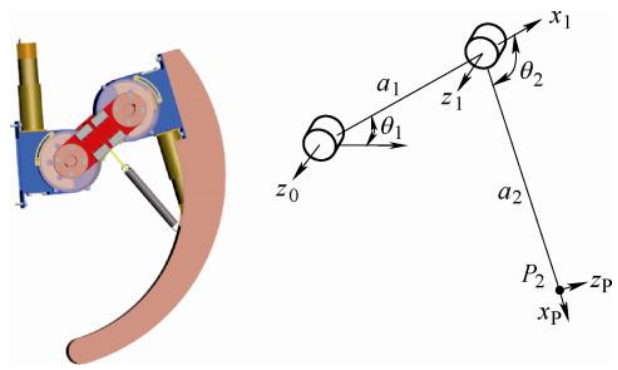

图 8 机器人 2 号腿 D-H 坐标系 
则以 2 号腿坐标系 $\left\{\Sigma_{20}\right\}$ 为参考坐标系, 足端点 $P_{2}$ 的位置 $\left(\theta_{1} 、 \theta_{2}\right.$ 是有符号的量)为

$$
\left\{\begin{array}{l}
x_{\mathrm{P}}=a_{1} \cos \theta_{1}+a_{2} \cos \left(\theta_{1}+\theta_{2}\right) \\
y_{\mathrm{P}}=a_{1} \sin \theta_{1}+a_{2} \sin \left(\theta_{1}+\theta_{2}\right) \\
z_{\mathrm{P}}=0
\end{array}\right.
$$

在机器人躯体坐标系 $\left\{\Sigma_{\mathrm{B}}\right\}$ 中, 2 号腿足端点 $P_{2}$ 的位置坐标满足关系

$$
\left[\begin{array}{llll}
{ }^{B} x_{P} & { }^{B} y_{P} & { }^{B} z_{P} & 1
\end{array}\right]={ }_{20}^{B} \boldsymbol{T} \times\left[\begin{array}{llll}
x_{P} & y_{P} & z_{P} & 1
\end{array}\right]
$$

根据式(3), 若给定足端点相对于躯体坐标系 $\left\{\Sigma_{\mathrm{B}}\right\}$ 位置, 由于 ${ }_{20}^{B} \boldsymbol{T}$ 是常量矩阵, 则躯体相对于腿 坐标系 $\left\{\Sigma_{20}\right\}$ 的位置亦可求得。根据式(2), 在已知足 端 $P_{2}$ 位置 $\left(x_{P} y_{P} z_{P}\right)$ 条件下, 求解各关节角度值, 得

$$
\begin{gathered}
\cos \theta_{2}=\frac{x_{p}^{2}+y_{p}^{2}-a_{1}{ }^{2}-a_{2}{ }^{2}}{2 a_{1} a_{2}} \\
\sin \theta_{2}= \pm \sqrt{1-\left(\cos \theta_{2}\right)^{2}}
\end{gathered}
$$

根据 $\operatorname{atan} 2$ 函数 ${ }^{[13]}$ 的定义

$$
\operatorname{atan} 2(y, x)=\arctan \left(\frac{y}{x}\right)+\frac{\pi}{2} \operatorname{sgn}(y)(1-\operatorname{sgn}(x))
$$

该函数可以返回一个 $(-\pi, \pi]$ 范围内的角度值, 且该 值具有唯一性, 因此本文用 $\theta=\operatorname{atan} 2(y, x)$ 来表示关 节角度解。则有

$$
\theta_{2}=\operatorname{atan} 2\left(\sin \theta_{2}, \cos \theta_{2}\right)
$$

根据 $\sin \theta_{2}$ 的符号, 给出两个解: $\sin \theta_{2} \geqslant 0$ 时, $\theta_{2,1} \in[0, \pi] ; \sin \theta_{2}<0$ 时, $\theta_{2,2} \in(-\pi, 0]$ 。

求解 $\theta_{1}$, 得

有

$$
\begin{aligned}
& \cos \theta_{1}=\frac{x_{p}\left(a_{1}+a_{2} \cos \theta_{2}\right)+y_{p} a_{2} \sin \theta_{2}}{a_{1}^{2}+a_{2}^{2}+2 a_{1} a_{2} \cos \theta_{2}} \\
& \sin \theta_{1}=\frac{y_{p}\left(a_{1}+a_{2} \cos \theta_{2}\right)-x_{p} a_{2} \sin \theta_{2}}{a_{1}^{2}+a_{2}^{2}+2 a_{1} a_{2} \cos \theta_{2}}
\end{aligned}
$$

$$
\theta_{1}=\operatorname{atan} 2\left(\sin \theta_{1}, \cos \theta_{1}\right)
$$

根据 $\theta_{2}$ 绕 $z_{1}$ 轴的正负号, 有两组解: $\left(\theta_{1}, \theta_{2,1}\right)$ 、 $\left(\theta_{1}, \theta_{2,2}\right)$ 。图 9 表示了 $\theta_{2}$ 的符号对应腿的不同形态。 $\theta_{2} \leqslant 0$ 选择逆解(1), 对应的解为 $\left(\theta_{1}, \theta_{2,2}\right)$ 。 $\theta_{2}>0$ 时 选择逆解(2), 对应的逆解为 $\left(\theta_{1}, \theta_{2,1}\right)$ 。而机器人 2 号 腿受布局限制只能相对躯体向前凸, 运动学逆解采 用逆解(1)。

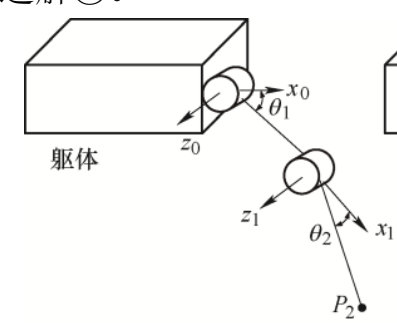

(a) 逆解 (1) $\theta_{2} \leqslant 0$

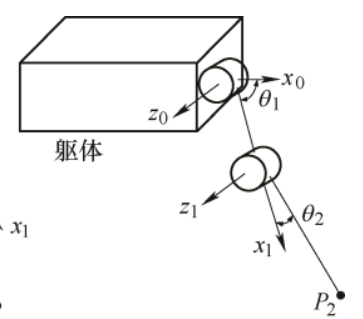

(b) 逆解 (2) $\theta_{2}>0$
图 9 机器人 2 号腿逆运动学两种解

\subsection{2 中腿运动学}

在分析中腿的位置关系时，以 4 号腿为例，可 以将其视为 3 自由度串联机械臂, 如图 10 所示。腿 三个连杆的长度分别为 $a_{3} 、 a_{4} 、 a_{5}$, 各个驱动关节 的角度为 $\theta_{i}(i=3,4,5)$, 关节角 $\theta_{3} 、 \theta_{4} 、 \theta_{5}$ 角度根 据右手定则确定，以右手拇指指向关节轴线的正方 向, 四指弯曲的方向为关节正方向。

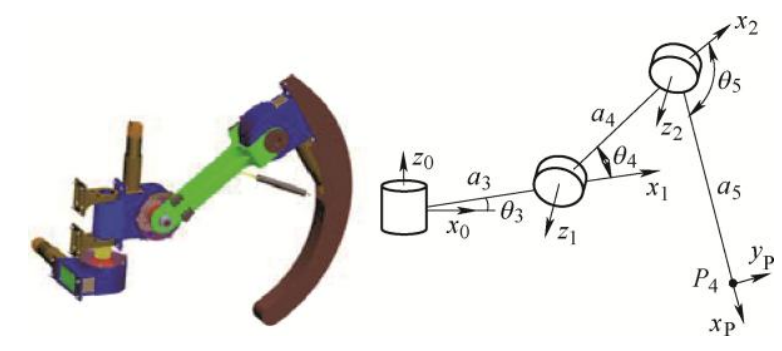

图 10 机器人 4 号腿 D-H 坐标系

足端点 $P_{4}$ 相对于 4 号腿坐标系 $\left\{\Sigma_{40}\right\}$ 的位置坐 标 $\left(\theta_{3} 、 \theta_{4} 、 \theta_{5}\right.$ 是有符号的量 $)$ 为

$$
\left\{\begin{array}{l}
x_{\mathrm{P}}=\cos \theta_{3}\left(a_{3}+a_{4} \cos \theta_{4}+a_{5} \cos \left(\theta_{4}+\theta_{5}\right)\right) \\
y_{\mathrm{P}}=\sin \theta_{3}\left(a_{3}+a_{4} \cos \theta_{4}+a_{5} \cos \left(\theta_{4}+\theta_{5}\right)\right) \\
z_{\mathrm{P}}=a_{4} \sin \theta_{4}+a_{5} \sin \left(\theta_{4}+\theta_{5}\right)
\end{array}\right.
$$

腿足端点 $P_{4}$ 在机器人躯体坐标系 $\left\{\Sigma_{\mathrm{B}}\right\}$ 的位置 坐标满足关系

$$
\left[\begin{array}{lllll}
{ }^{B} x_{P} & { }^{B} y_{P} & { }^{B} z_{P} & 1
\end{array}\right]^{\mathrm{T}}={ }_{40}^{B} \boldsymbol{T} \times\left[\begin{array}{llll}
x_{P} & y_{P} & z_{P} & 1
\end{array}\right]^{\mathrm{T}}
$$

根据式(7), 在给定足端点 $P_{4}$ 相对于躯体坐标系 $\left\{\Sigma_{\mathrm{B}}\right\}$ 位置条件下, 由于 ${ }_{40}^{B} \boldsymbol{T}$ 是常量矩阵, 则足端点 $P_{4}$ 相对于腿坐标系 $\left\{\Sigma_{40}\right\}$ 的位置亦可求得。根据式(6), 在已知足端坐标系 $\left(x_{P} y_{P} z_{P}\right)$ 条件下, 求解各关节角 度值。推导得

$$
\theta_{3}=\arctan 2\left(y_{P}, x_{P}\right)
$$

定义过程参数

$$
m=\left(x_{P}-a_{3} \cos \theta_{3}\right)^{2}+\left(y_{P}-a_{3} \sin \theta_{3}\right)^{2}
$$

从而有

$$
\cos \theta_{5}=\frac{m+z_{p}^{2}-a_{4}^{2}-a_{5}^{2}}{2 a_{4} a_{5}} \sin \theta_{5}= \pm \sqrt{1-\left(\cos \theta_{5}\right)^{2}}
$$

故有

$$
\theta_{5}=\arctan 2\left(\sin \theta_{5}, \cos \theta_{5}\right)
$$

根据 $\sin \theta_{5}$ 的符号, 给出两个解

$$
\theta_{5,1} \in[0, \pi] \quad \theta_{5,2}=-\theta_{5,1} \in[-\pi, 0]
$$

对 $\theta_{4}$ 求解

$$
\begin{aligned}
& \cos \theta_{4}=\frac{\sqrt{m}\left(a_{4}+a_{5} \cos \theta_{5}\right)+z_{P} a_{5} \sin \theta_{5}}{a_{4}^{2}+a_{5}^{2}+2 a_{4} a_{5} \cos \theta_{5}} \\
& \sin \theta_{4}=\frac{z_{P}\left(a_{4}+a_{5} \cos \theta_{5}\right)-\sqrt{m} a_{5} \sin \theta_{5}}{a_{4}^{2}+a_{4}^{2}+2 a_{4} a_{5} \cos \theta_{5}}
\end{aligned}
$$


因此 $\theta_{4}$ 的解为

$$
\theta_{4}=\arctan 2\left(\sin \theta_{4}, \cos \theta_{4}\right)
$$

综上, 4 号腿存在 2 组运动学逆解 $\left(\theta_{3}, \theta_{4}, \theta_{5,1}\right)$ 、 $\left(\theta_{3}, \theta_{4}, \theta_{5,2}\right)$, 其对应的形态如图 11 所示。

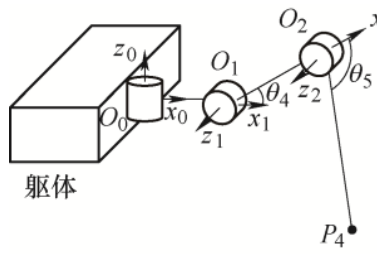

(a) 逆解 (1) $\theta_{5} \leqslant 0$

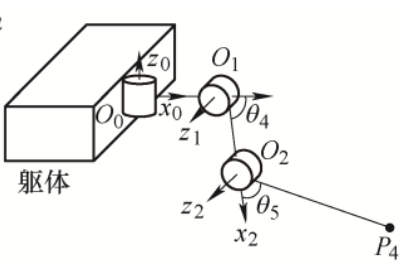

(b) 逆解 (2) $\theta_{5}>0$
图 11 机器人 4 号腿的两种运动学逆解

根据逆解中 $\theta_{5}$ 的取值范围可以确定所对应的 逆解。 $\theta_{5} \leqslant 0$ 结构 (1), 逆解为 $\left(\theta_{3}, \theta_{4}, \theta_{5,2}\right) ; \theta_{5}>0$, 结构(2), 逆解为 $\left(\theta_{3}, \theta_{4}, \theta_{5,1}\right)$ 。根据图 11 表示 $\theta_{5}$ 的符 号对应腿的不同形态, 而 4 号腿受布局限制只能相 对躯体向外凸, 运动学逆解采用逆解 (1), 逆解为 $\left(\theta_{3}, \theta_{4}, \theta_{5,2}\right)$ 。

\subsection{3爬行模式直行}

机器人爬行模式直行是机器人的基础运动功能, 直行步态的设计直接影响到机器人能耗和灵活性等 能力, 因此选择合适的步态至关重要。尽管六足机 器人的步态模式中包含四足步态和五足步态, 但相 关统计表明三足步态适用于大部分崎岖地 形 $^{[14]}$, 只有在六足机器人受到未知地形和自身运动状态的 严重约束才会采用四足步态和五足步态, 以便于穿 过运动受限的空间, 因此本文基于三足步态对机器 人步行直线运动进行研究。

当机器人采用三足步态时, 编号 $1 、 4 、 5$ 腿为 一组, 编号 $2 、 3 、 6$ 腿为一组。图 13 表示机器人三 足步态直行前进过程, 在 $1 、 4 、 5$ 作为支撑腿时, 支撑腿推动躯体前移， $2 、 3 、 6$ 腿作为摆动腿向前 迈步, 且两组腿在支撑相和摆动相之间不断切换,

图 12 为机器人以三足步态直行过程。

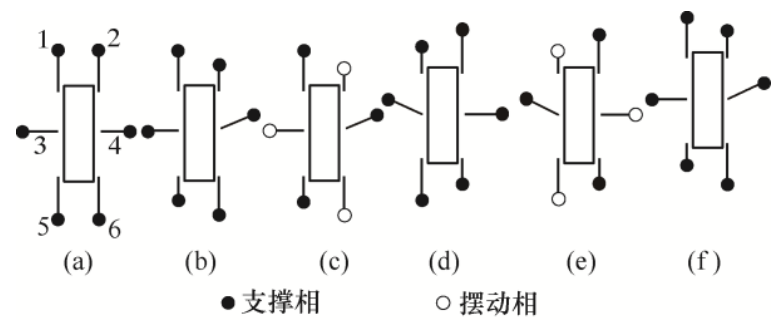

图 12 爬-滚机器人直行步态图

为减少内力对抗, 保证机器人支撑腿与地面不 会产生滑移, 对机器人足端进行轨迹规划 ${ }^{[15]}$ 。如图 13 所示, 根据爬行腿处于支撑相和摆动相分为足端
支撑相轨迹和足端摆动相轨迹, $t_{0}$ 时刻表示摆动相 的起点, $t_{1}$ 时刻表示摆动相的路径点, $t_{2}$ 时刻表示 摆动相终点和支撑相的起点， $t_{3}$ 时刻表示支撑相的 终点。

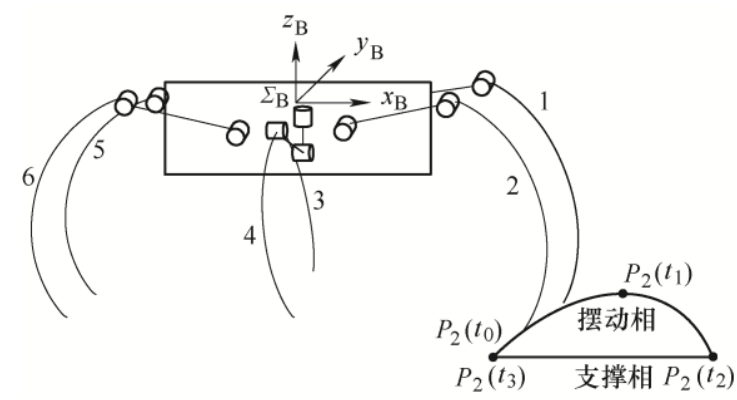

图 13 爬-滚机器人直行过程中的足端轨迹规划

在常用轨迹曲线中，多项式拟合曲线不仅宽高 比、起始角落地角可以通过设定边界条件而改变, 还可以通过设定其一阶、二阶导数, 以保证其速度 加速度连续 ${ }^{[16]}$, 因此本文选用多项式轨迹曲线对机 器人直行运动足端轨迹进行规划。

在图 13 中, 对于摆动腿, 从 $t_{0} \rightarrow t_{1} \rightarrow t_{2}$ 为爬行 模式的摆动相运动轨迹。在摆动相轨迹中, 起始点 时刻 $t_{0}$ 对应足端点位置 $P_{0}$ 、速度 $v_{0}$ 、加速度 $a_{0}$, 落 脚点时刻 $t_{2}$ 对应足端点位置 $P_{2}$ 、速度 $v_{2}$ 、加速度 $a_{2}$, 路径点时刻 $t_{1}$ 对应足端点位置 $P_{1}$ 。则摆动相轨迹为

$$
P(t)=\sum_{i=0}^{6} b_{i}\left(t-t_{0}\right)^{i}
$$

7 个边界条件为: 摆动相起始时刻 $t_{0}$ 对应的足 端位置 $P\left(t_{0}\right)=\left[\begin{array}{lll}x_{0} & y_{0} & z_{0}\end{array}\right]$, 速度 $\dot{P}\left(t_{0}\right)$, 加速度 $\ddot{P}\left(t_{0}\right)$; 路径点时刻 $t_{1}$ 对应的位置 $P\left(t_{1}\right)=\left[\begin{array}{lll}x_{1} & y_{1} & z_{1}\end{array}\right]^{\mathrm{T}}$; 落地时 刻 $t_{3}$ 对应的足端位置 $P\left(t_{2}\right)=\left[\begin{array}{lll}x_{2} & y_{2} & z_{2}\end{array}\right]^{\mathrm{T}}$, 速度 $\dot{P}\left(t_{2}\right)$, 加速度 $\ddot{P}\left(t_{2}\right)$ 。通过设定上述边界条件可以得出摆动 相足端轨迹。

在图 13 中, 爬行腿在摆动相结束后切换为支撑 相, 其足端点相对于地面静止。机器人躯体采用五 次多项式曲线进行前向运动, 从而支撑腿相对于躯 体质心的运动轨迹为

$$
x_{\mathrm{B}}(t)=\sum_{i=0}^{5} c_{i}\left(t-t_{2}\right)^{i} \quad y_{\mathrm{B}}(t)=0 \quad z_{\mathrm{B}}(t)=0
$$

6 个边界条件为: 支撑相起始时刻 $t_{2}$ 对应的足 端位置 $P\left(t_{2}\right)=\left[\begin{array}{lll}x_{2} & y_{2} & z_{2}\end{array}\right]^{\mathrm{T}}$, 速度 $\dot{P}\left(t_{2}\right)$, 加速度 $\ddot{P}\left(t_{2}\right)$; 支撑相结束时刻 $t_{3}$ 对应的足端位置 $P\left({ }_{3} t\right)\left[{ }_{0}^{x} \quad e^{\mathrm{T}}\right.$, 速度 $\dot{P}\left(t_{3}\right)$, 加速度 $\ddot{P}\left(t_{3}\right)$ 。通 过设定上述边界条件可以得出躯体质心运动轨迹。

通过式(11)、(12)规划出机器人足端点运动学轨 迹, 将足端轨迹曲线方程代入运动学逆解中, 则可 
求得机器人爬行模式下各腿关节角度变化值。

\subsection{4爬行模式原地转向}

机器人的转向包括前进过程中转向和原地转 向 $^{[17]}$, 相比前进转向, 原地转向使机器人具有更灵 活的机动性。图 14 表示机器人原地右转过程各腿的 运动步态 ${ }^{[18]}$, 原地转向摆腿顺序: 中腿支撑躯体抬 升 $\rightarrow$ 躯体转向 $\rightarrow$ 中腿放下躯体 $\rightarrow$ 中腿复位。
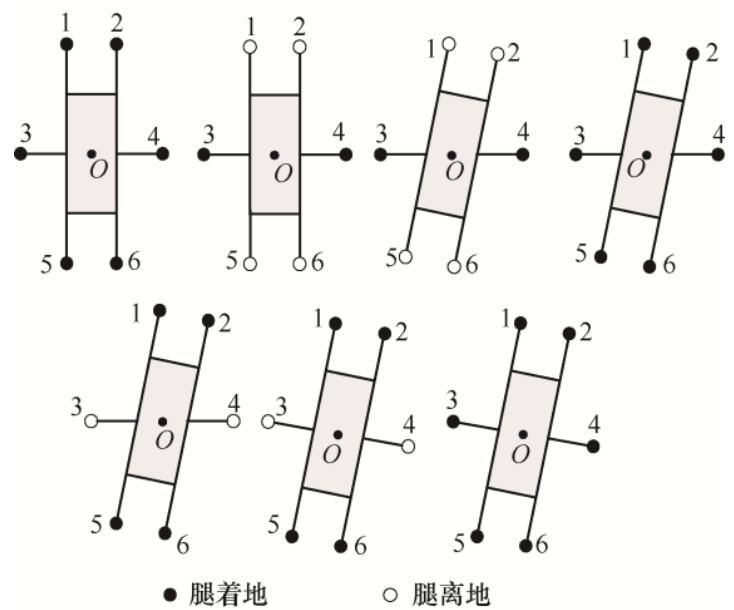

图 14 机器人原地转向步态

图 15 表示 4 号腿抬升躯体 $h$ 高度过程, 及对应 过程的起点时刻 $t_{0}$ 和终点时刻 $t_{1}$ 。以躯体坐标系 $\left\{\Sigma_{\mathrm{B}}\right\}$ 为参考, 足端点相对于躯体坐标系下降 $h$ 高度。

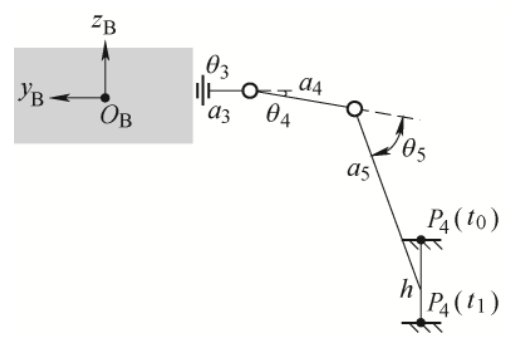

图 15 原地转向过程抬升躯体

图 15 中, 在躯体坐标系 $\left\{\Sigma_{\mathrm{B}}\right\}$ 下, 足端 $P_{4}$ 从 $t_{0}$ 至 $t_{1}$ 时刻轨迹变化可描述为

$$
x(t)=x\left(t_{0}\right), y(t)=y\left(t_{0}\right), z(t)=z\left(t_{0}\right)-\frac{t-t_{0}}{t_{1}-t_{0}} h
$$

图 16 表示机器人在中腿支撑下的转向, 及对应 的起点时刻 $t_{1}$ 和终点时刻 $t_{2}$, 机器人躯体转向 $\omega$ 过 程, 中腿足端点 $P_{3} 、 P_{4}$ 位置相对地面保持静止, 相 对机器人质心坐标系 $\left\{\Sigma_{\mathrm{B}}\right\}$ 为一段圆弧形轨迹, 设 $R_{C}$ 为该轨迹圆弧的半径。

以躯体坐标系 $\left\{\Sigma_{\mathrm{B}}\right\}$ 为参考, 从 $t_{1}$ 至 $t_{2}$ 时刻躯体 转向过程, 中腿足端点 $P_{4}$ 轨迹曲线为

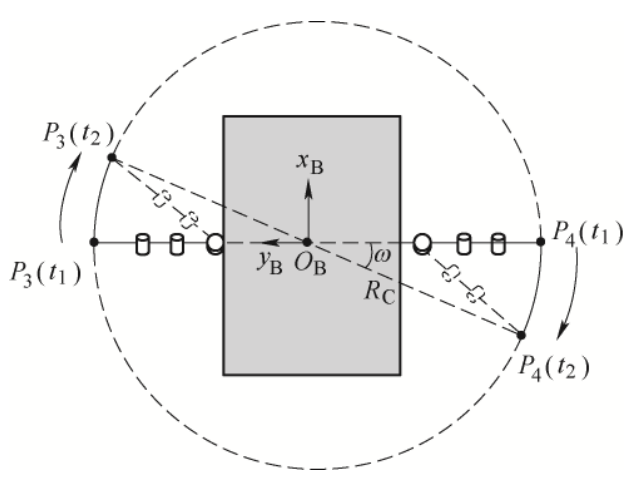

图 16 原地转向过程躯体转向

$$
\left\{\begin{array}{l}
x(t)=-R_{C} \sin \omega \\
y(t)=-R_{C} \cos \omega \\
z(t)=z\left(t_{1}\right)
\end{array}\right.
$$

图 17 表示完成躯体转向后降低躯体高度至前 后腿支撑躯体，及对应的起点时刻 $t_{2}$ 和终点时刻 $t_{3}$, 此为躯体抬升的逆过程。

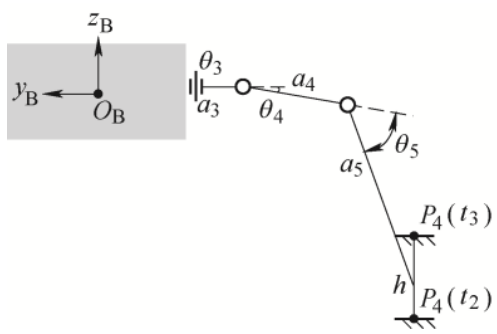

图 17 原地转向过程降低躯体高度

图 17 中, 以躯体坐标系 $\left\{\Sigma_{\mathrm{B}}\right\}$ 为参考, 足端 $P_{4}$ 在 $t_{2}$ 至 $t_{3}$ 时刻轨迹曲线为

$$
x(t)=x\left(t_{2}\right), y(t)=y\left(t_{2}\right), z(t)=z\left(t_{2}\right)+\frac{t-t_{2}}{t_{3}-t_{2}} h
$$

将式(13) (15)代入中腿运动学逆解中, 可求得 机器人在原地转向各运动阶段机器人中腿各关节角 度值。完成图 15１7 所示 3 个阶段后，复位中腿回 到原地转向初始时刻相对躯体位置，完成一个周期 的原地转向步态。

\section{2 滚动模式运动分析}

机器人主要在平地或下坡路段采用滚动模式, 以实现快速通过性能。滚动模式下能攀爬的最大斜 坡角度和越过障碍物的最大高度是一项重要的性能 评价指标。根据辅助腿的工作原理, 辅助腿机构通 过快速拨动连杆给予处于滚动模式状态的机器人一 个向前滚动的初速度。因此, 躯体滚动的初速度是 由连杆的拨动快慢决定的, 布置在辅助腿组件上的 电动机转速影响着机器人滚动时的初速度。

\section{2 .1 平地工况}

图 18 为平坦路面上机器人滚动模式的受力分 
析, 机器人受辅助腿的推动力 $F_{\mathrm{T}}$ 、重力 $G$ 、地面的 支撑力 $F_{\mathrm{N}}$ 和摩擦力 $f, \mu_{1}$ 表示辅助腿与地面的静摩 擦系数, $\mu_{2}$ 表示机器人滚动模式 “轮”与地面的静摩 擦系数, 设滚动点为 $O$ 点, $l$ 表示辅助腿施力点与 滚动点之间的距离, $d$ 为质心在重力方向与滚动点 之间的距离, $\alpha$ 为辅助腿与地面的夹角。

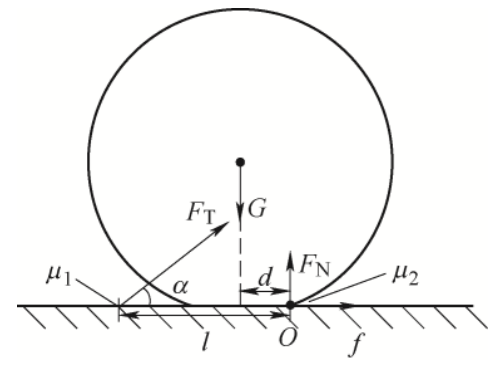

图 18 平坦工况滚动模式受力分析

为保证辅助腿与地面不发生相对滑移, 保证辅 助腿良好的驱动功能, 辅助腿应满足以下条件

$$
F_{\mathrm{T}} \cos \alpha \leqslant \mu_{1} F_{\mathrm{T}} \sin \alpha
$$

因此为保证机器人辅助腿更好驱动机器人滚动, 可通过改变足端表面形貌, 以增大与地的接触摩擦 系数。由式(16)可得出在 $\alpha \geqslant \operatorname{arccot} \mu_{1}$ 时, 辅助腿的 驱动功能有效。

当驱动力矩大于重力产生的阻碍力矩后, 球体 发生滚动。即

$$
F_{\mathrm{T}} l \sin \alpha \geqslant G d
$$

\subsection{2爬坡工况}

最大爬坡角是衡量机器人滚动模式爬坡能力的 一个重要指标, 机器人的爬坡能力除受到外部条件 (斜坡角度以及斜坡地面状况)的限制, 还要受到机 器人自身的机械结构的影响。本文分析以机器人不 离地为基本约束, 以确保机器人与地面保持接触。

图 19 为机器人在爬坡过程中的受力分析, 其主 要受力有重力 $G$ 、斜坡支持力 $F_{\mathrm{N}}$ 、摩擦力 $f$ 、辅助 腿的推动力 $F_{\mathrm{T}}, \mu_{1}$ 表示辅助腿与地面的静摩擦因数, $\mu_{2}$ 表示机器人滚动模式 “轮”与地面的静摩擦因数。 设滚动点为 $O, l$ 表示辅助腿施力点与滚动点之间的 距离, $R_{\mathrm{W}}$ 为机器人 “轮” 的半径, $\alpha$ 为辅助腿与地 面夹角, $\beta$ 为斜坡角度。

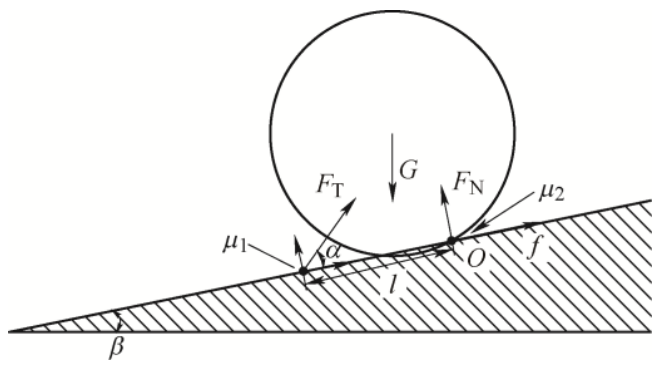

图 19 爬坡工况机器人滚动模式受力分析
辅助腿足端不发生打滑是确保机器人不会向下 滚动或带来较大冲击的重要因素 ${ }^{[19]}$ 。即在 $\alpha \geqslant \operatorname{arccot} \mu_{1}$ 条件下, 能保证机器人良好的推动效 果 (不打滑), 且在完成推动后机器人辅助腿起到良 好的支撑作用。

在斜坡滚动结束后, 机器人辅助腿处于图 $6 \mathrm{a}$ 所示的就位状态，防止机器人在斜坡上回滚。在辅 助腿就位状态, 辅助腿可提供机器人支持力, 机器 人重心的垂直线应位于辅助腿支撑点与足支撑点之 间, 设此时能越过的临界爬坡角度为 $\beta_{1}$, 则有

$$
\tan \beta_{1}=\frac{l}{R_{\mathrm{w}}}
$$

从而

$$
\beta_{1}=\arctan \frac{l}{R_{\mathrm{W}}}
$$

对三维模型测量的值代入上式中, 得机器人不 回滚的临界坡度角

$$
\beta_{1}=45^{\circ}
$$

当斜面坡度角过大时, 机器人爬坡过程中会出 现“打滑”现象。假设机器人刚出现打滑的斜坡临界 坡度角度为 $\beta_{2}$, 轮与斜面接触的静摩擦系数为 $\mu_{2}$ 。 则可以推出重力的斜面分量打破静摩擦力产生滑动 现象的临界值关系式

$$
\begin{gathered}
G \sin \beta_{2}=\mu_{2} G \cos \beta_{2} \\
\beta_{2}=\arctan \mu_{2}
\end{gathered}
$$

设 $\mu_{2}=0.6$, 在不考虑辅助腿的作用因素, 仅 考虑机器人与地的静摩擦情况, 机器人的理论最大 爬坡角度 $\beta_{2}=30.96^{\circ}$ 。

考虑到辅助腿的作用, 因机器人的初速度是由 辅助腿提供, 在惯性力作用下继续向前滚动, 因此 需考虑辅助腿能给与的最大初速度 $v_{\text {max }}$ 。为保证为 保持机器人滚动的连续性, 机器人在初速度下应至 少滚动一个周期, 此时斜面坡度角设为 $\beta_{3}$, 则机器 人滚动的最大初速度 $v_{\max }$ 应满足条件

$$
\frac{1}{2} m v_{\max }^{2} \geqslant m g\left(2 \pi R_{\mathrm{W}} \sin \beta_{3}\right)
$$

则有

$$
\beta_{3} \leqslant \arcsin \frac{v_{\max }^{2}}{4 g \pi R_{\mathrm{W}}}
$$

机器人的最大爬坡角度

$$
\beta=\min \left\{\beta_{1}, \beta_{2}, \beta_{3}\right\}
$$

推动机器人前向滚动所需辅助腿的推动力产生 的力矩应能克服阻力, 即 


$$
F_{\mathrm{T}} l \sin \alpha \geqslant G R_{\mathrm{w}} \sin \beta
$$

从而

$$
F_{\mathrm{T}} \geqslant \frac{G R_{\mathrm{W}} \sin \beta}{l \sin \alpha}
$$

\subsection{3 越障工况}

机器人在滚动模式中可借助辅助腿, 推动机器 人完成越障。为保证机器人良好的越障性能, 机器 人 “轮” 在障碍物处无相对滑动。图 20 为机器人遇 障时的受力分析, 其主要受力有重力 $G$ 、障碍物支 持力 $F_{\mathrm{N}}$ 、摩擦力 $f$ 、辅助腿的推动力 $F_{\mathrm{T}}, \mu_{1}$ 表示 辅助腿与地面的静摩擦系数, $\mu_{2}$ 表示机器人 “轮” 与地面的静摩擦系数, 越障高度为 $h$, 障碍物接触 点和机器人 “轮” 心连线与重力加速度方向的夹角 为 $\gamma, R_{\mathrm{w}}$ 为机器人 “轮” 的半径, $\alpha$ 为辅助腿与地面 夹角, 越障时滚动体与地面的接触点由 $O_{1}$ 变为 $O_{2}$ 。

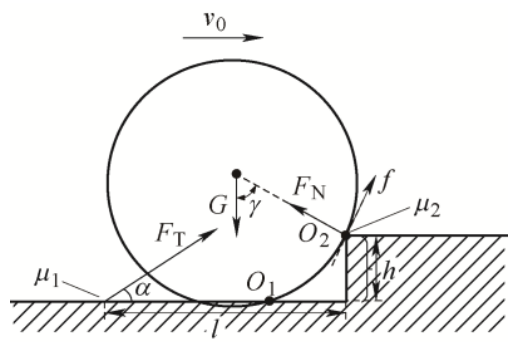

图 20 越障工况机器人滚动模式受力分析

“轮” 不打滑通过路面的条件 ${ }^{[20]}$

$$
\mu_{2}>\tan \gamma
$$

则在 “轮” 不打滑的情况下, 机器人滚动模式最大 越障高度

$$
h=R_{\mathrm{w}}-R_{\mathrm{W}} \cos \gamma
$$

辅助腿施加的力应能克服机器人重力产生的阻 力矩, 则机器人依靠辅助腿推动越障所需辅助腿驱 动力大小为

$$
F_{\mathrm{T}} \geqslant \frac{G R_{\mathrm{W}} \sin \gamma}{l \sin \alpha-h \cos \alpha}
$$

在 $\alpha \geqslant \operatorname{arccot} \mu_{1}$ 条件下, 辅助腿的推动功能有 效, 在机器人滚动模式越障过程中, $\alpha$ 角不断增大, 故此处不考虑辅助腿的打滑情况。

机器人翻越凸台障碍过程中, 滚动摩擦点从 $O_{1}$ 突变到 $O_{2}$, 越障过程中机器人的轨迹变化如图 21 所示。

取轮与地面的最大静摩擦系数为 $\mu_{2}=0.6$, 计 算出机器人滚动模式不打滑的最大越障高度为 $32.06 \mathrm{~mm}$ 。该方法仅考虑机器人在摩擦作用下能跨 越的最大障碍高度, 实际应用中, 可以改变 “轮”
表面纹理, 利用机器人 “轮” 与地面产生的剪切力, 增大轮的抓地力, 增加最大越障高度, 理论上通过 改变轮的表面纹理形状可增大机器人越障高度至 “轮” 半径长度。

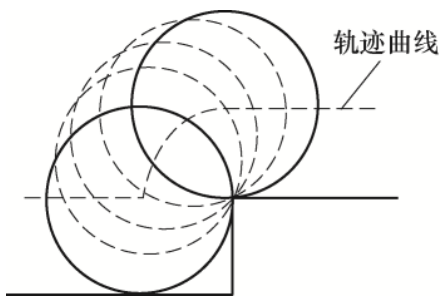

图 21 机器人滚动模式越障质心变化轨迹

\section{3 基于 ADAMS 的运动仿真}

在 ADAMS View 仿真环境中, 导入 Pro/E 三维模 型, 添加足部与地面的接触(Contact), 接触力采用冲 击函数模型(IMPACT), 取接触刚度 $k=1000 \mathrm{~N} / \mathrm{mm}$, 形状指数 $e=2.2$, 最大允许穿透深度 $d_{\text {max }}=1.5 \mathrm{~mm}$, 阻尼值 $c=200$, 机器人与地为库仑摩擦, 静摩擦系 数为 0.6 , 动摩擦系数为 0.5 。

\section{1 爬行模式仿真}

\subsection{1 直行仿真}

仿真中机器人的六条腿以给定角度的方式进行 运动, 以表 2 为机器人直行的约束条件, 规划出足 端点运动轨迹, 结合运动学逆解, 计算出机器人前 进过程中各腿关节的角度值。

表 2 直行过程主要运动参数

\begin{tabular}{lc}
\hline \multicolumn{1}{c}{ 参数 } & 数值 \\
\hline 质心离地高度 $/ \mathrm{mm}$ & 192 \\
摆动相前进位移 $/ \mathrm{mm}$ & 100 \\
摆动相跨越高度 $/ \mathrm{mm}$ & 35 \\
支撑相位移 $/ \mathrm{mm}$ & 100 \\
\hline
\end{tabular}

将求得的腿关节角度值作为 ADAMS 仿真中腿 关节的驱动条件, 得到图 22 机器人直行过程。为提 高仿真的精度, 共仿真 5 个周期的三角前进步态。 图 22a、22b 为机器人三足爬行步态的准备阶段, 图 $22 \mathrm{c} \sim 22 \mathrm{f}$ 为 $1 、 4 、 5$ 号腿和 $2 、 3 、 6$ 号腿交替运动, 推动机器人前进阶段。

从 $\mathrm{ADAMS}$ 后处理图像中可以得出机器人运动 过程中质心轨迹运动变化, 图 23 为质心在 $x 、 y 、 z$ 方向上的位移变化量, 机器人刚开始运动时, 有一 段调整过程, 质心略微降低, 随后机器人以三角步 态向前移动。 


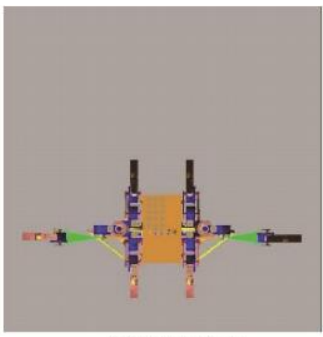

(a)初始状态

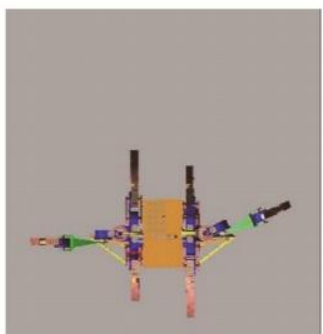

(c)1、4、5 腿摆动, 2、 3、6 支撑

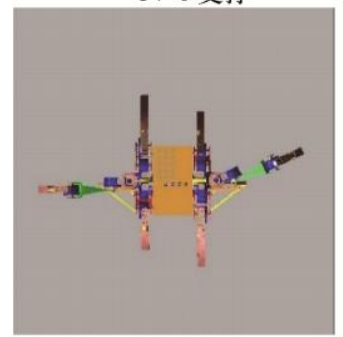

(e) 1、4、5 腿摆动，2、3、 6 支撑

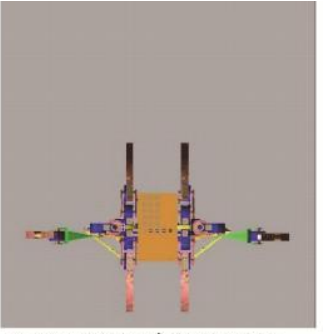

(b)六腿朝远离躯体调整, 直到躯体达到目标高度

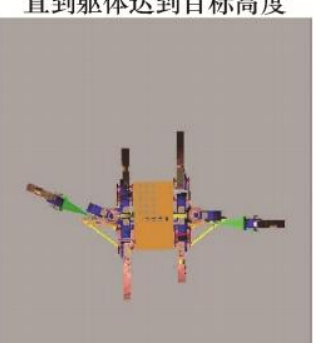

(d) 1、4、5 腿摆动，2、 3、6 支撑

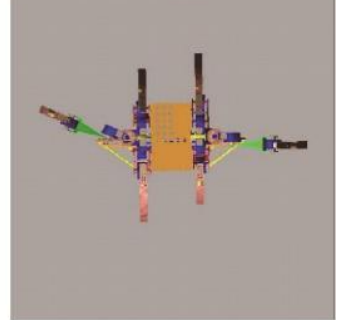

(f) 2、3、6 腿摆动，1、

$4 、 5$ 支撑

图 22 机器人三足步态前进过程

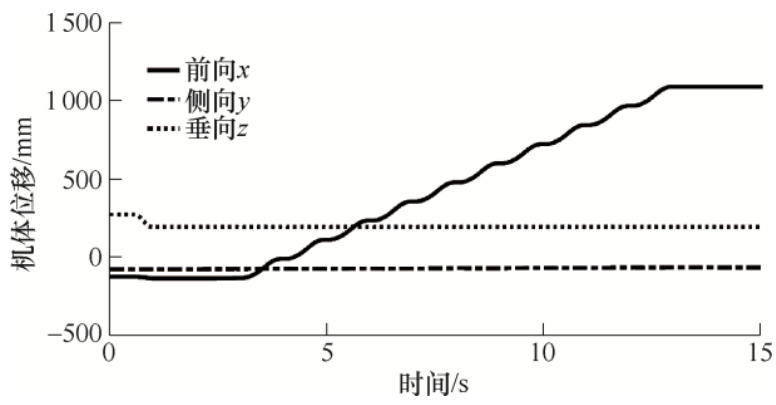

图 23 机器人直行仿真中躯体质心位移变化

根据图 23 仿真曲线, 得出机器人一个周期的前 行距离

$$
s=191 \mathrm{~mm}
$$

侧向滑移量

$$
b=2 \mathrm{~mm}
$$

滑移率

$$
\varepsilon=1.05 \%
$$

仿真结果表明机器人在直行过程中具有较高的 直线度, 侧向滑移较小, 表明基于足端轨迹规划的 直行步态设计合理。

\subsection{2 原地转向仿真}

机器人在原地转向过程中, 先抬升躯体从高度 $185 \mathrm{~mm}$ 至 $215 \mathrm{~mm}$, 随后机器人躯体转动 $30^{\circ}$, 再
将躯体从高度 $215 \mathrm{~mm}$ 调整至 $185 \mathrm{~mm}$, 依靠前后腿 支撑躯体, 最后中腿回到初始状态。根据式(13) (15) 完成足端轨迹的计算, 并将足端轨迹代入逆运动学 中, 求得机器人原地转向步态中各腿关节角度值。 将求得的角度值导入到 ADAMS 关节驱动函数中, 得到图 24 所示原地转向仿真运动过程。

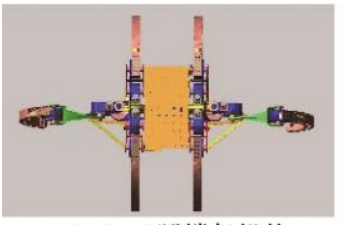

(a) 3、4腿撑起机体

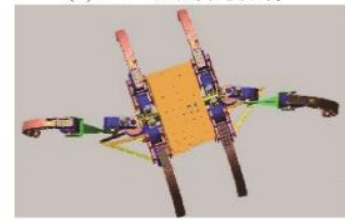

(c) 放下躯体

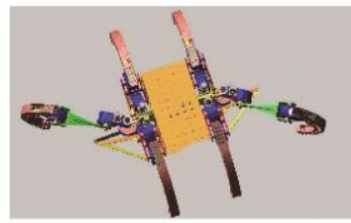

(b) 3、4腿撑起身体后转向

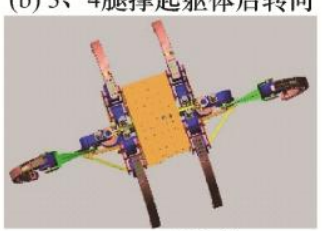

(d) 3、4腿复位
图 24 机器人原地转向仿真过程

为便于测量, 定义仿真过程中腿足端点的连线 与初始连线的夹角为躯体转角变化值, 在原地转向 步态中躯体转角变化如图 25 所示。

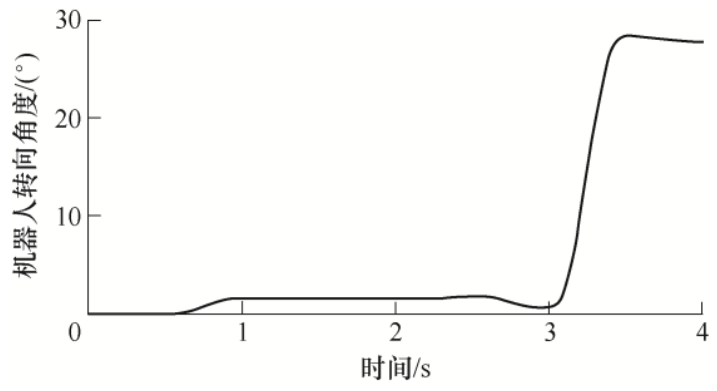

图 25 机器人相对初始位置转角变化

爬-滚机器人转向过程中设定的转向 $30^{\circ}$, 仿真 值为 $27.76^{\circ}$, 比理论值减少了 $7 \%$ 。

爬-滚机器人爬行模式仿真值与理论值的比较 见表 3 。理论值与仿真值的差距来源是因为机器人 在爬行模式中, 足端与地面存在摩擦滑移。此外, 机器人足端是球形结构, 理论值是按足端尖点进行 计算, 腿的长度与理论值计算值存在差距。

表 3 爬行模式仿真与理论对比

\begin{tabular}{lcc}
\hline \multicolumn{1}{c}{ 运动参数 } & 理论值 & 仿真值 \\
\hline 爬行模式直行一个周期步长 $/ \mathrm{mm}$ & 200 & 192 \\
爬行模式直行一个周期侧移量 $/ \mathrm{mm}$ & 0 & 2 \\
爬行模式原地转向角度 $/\left(^{\circ}\right)$ & 30 & 27.76 \\
\hline
\end{tabular}

\section{2 滚动模式仿真}

\subsection{1 平坦地面滚动仿真}

在水平地面上, 不考虑滑动摩擦和能量耗散, 理论上机器人只需一定的初速度能大于克服重力阻 
力矩做功, 机器人就能滚动。将 $\mathrm{Pro} / \mathrm{E}$ 中处于滚动 模式状态的机器人模型导入 ADAMS 环境中, 设置 “轮”与地面接触条件, 添加辅助腿机构的驱动力矩, 得到机器人在平坦地面上滚动过程如图 26 所示。

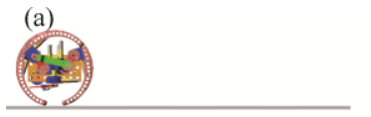

(c)

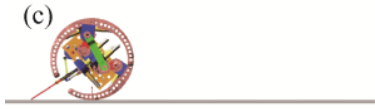

(e)

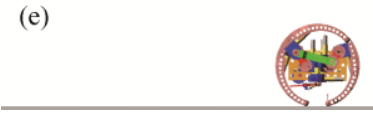

(f)

图 26 机器人平地滚动过程

在 ADAMS 后处理环境中, 得出机器人在 $x 、 y$ 、 $z$ 方向上的位移变化曲线见图 27 。

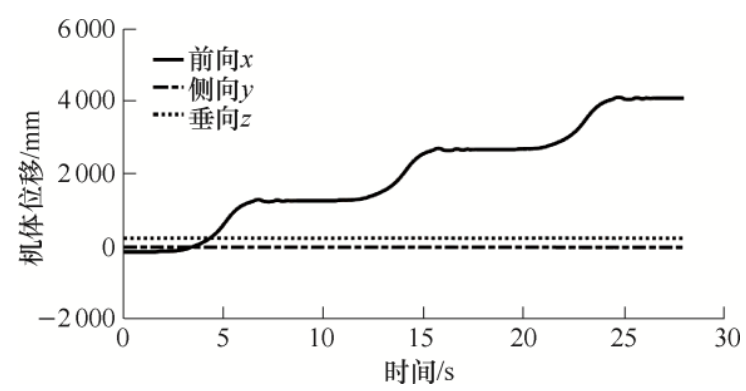

图 27 平地工况下机器人滚动模式质心位移变化

从图 27 可以得出, 机器人在平地工况下质心位 移变化量曲线平滑, 在机器人滚动 3 个周期中, 前 进方向的位移 $4 \mathrm{~m}$, 侧向偏移量在 $5 \mathrm{~mm}$ 内, 质心在 $z$ 轴方向有轻微波动。

机器人辅助腿提供的推动力变化如图 28 所示, 从图中可以得出推动机器人滚动的最大推动力约为 $115 \mathrm{~N}$, 具有较大的冲击, 分析得推动力最大时刻为 机器人辅助腿刚触地推动机器人滚动时刻。

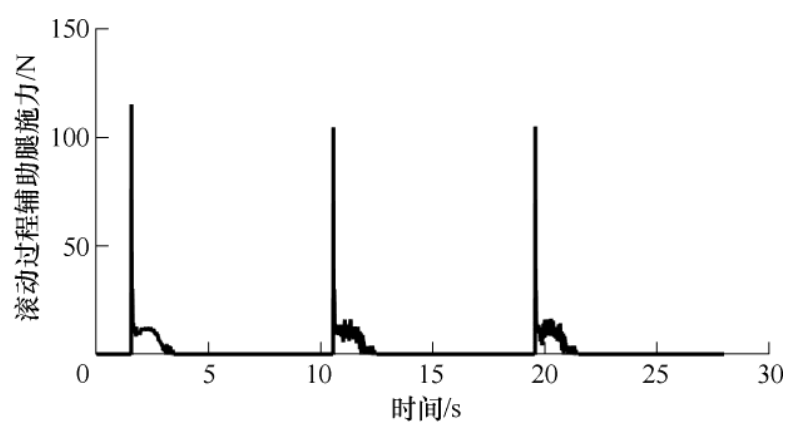

图 28 机器人辅助腿的推动力

\subsection{2 滚动模式爬坡仿真}

在滚动模式爬坡运动仿真中, 经多次测试, 辅 助腿能推动机器人在斜坡上滚动的最大初速度约为

$1.5 \mathrm{~m} / \mathrm{s}$ 左右, 若超过该速度, 则会因为辅助腿施力 过大、机器人滚动速度过快造成运动不平稳, 根据 式(20)、(21), 机器人的最大爬坡角约为 $4.6^{\circ}$ 。在仿 真中多次调整爬坡角, 在机器人平稳爬坡条件下, 测得机器人跨越的斜坡角度约为 $5^{\circ}$, 攀爬斜坡过程 如图 29 所示, 共仿真了 3 个滚动周期。

(a)

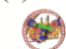

(c)

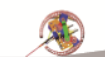

(e)

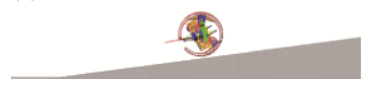

图 29 机器人爬坡滚动

从 ADAMS 仿真结果中得出机器人爬坡运动过 程中质心速度变化如图 30 所示, 可得出最大速度约 为 $1.55 \mathrm{~m} / \mathrm{s}$ 。

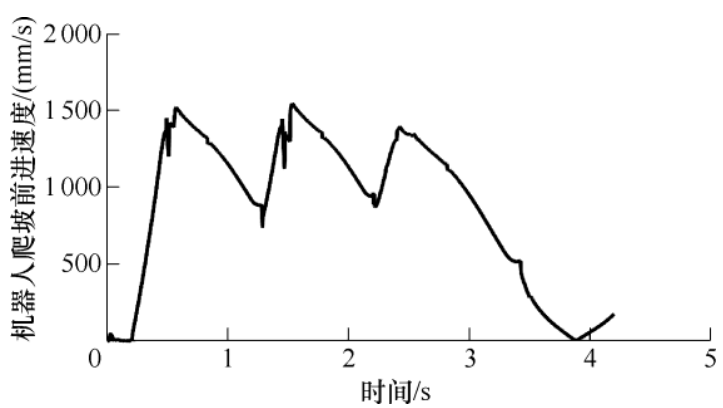

图 30 机器人爬坡过程中质心速度大小变化

辅助腿足端与地面接触力的变化如图 31 所示。 在辅助腿刚触地时, 有较短时间的力突变, 在推动 机器人滚动过程中平均施力大小约为 $70 \mathrm{~N}$ 。

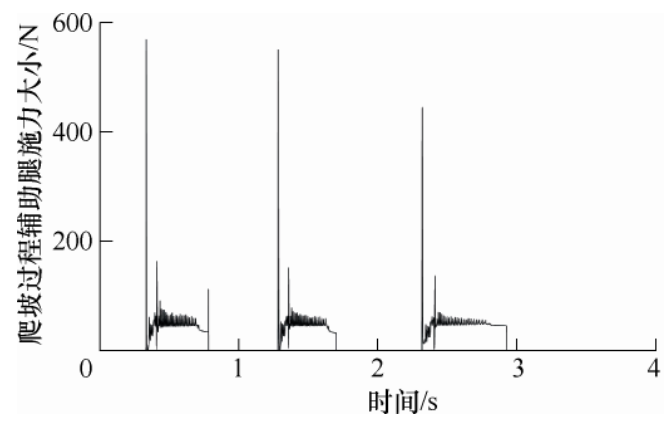

图 31 辅助腿足端与地面接触力的变化

从图 30 可以得出, 辅助腿能推动机器人以最大 初速度 $1.55 \mathrm{~m} / \mathrm{s}$ 越过 $5^{\circ}$ 的斜坡。从图 31 仿真分析 中爬-滚机器人在辅助腿刚触地时有较大的冲击, 随 后以相对较平缓的力推动机器人爬坡滚动。 


\subsection{3 滚动模式越障仿真}

根据机器人越障性能的分析, 理论上不断增加 静摩擦系数, 提高辅助腿的驱动力矩, 机器人能越 过的凸台障碍高度变大。根据式(23)、(24), 在机器 人 “轮” 与地的静摩擦系数 $\mu_{2}=0.6$ 条件下, 计算 得机器人最大越障高度 $h=32.06 \mathrm{~mm}$ 。通过多次调 整越障高度, 添加辅助腿电动机的驱动力矩, 测得 机器人滚动模式下能越过的障碍高度约为 $32 \mathrm{~mm}$, 得到如图 32 所示爬-滚机器人越障过程。

(a)

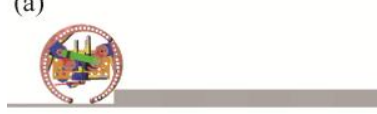

(c)

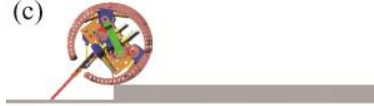

(e)

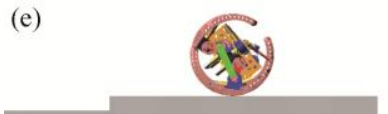

(f)

图 32 机器人滚动模式越障

在机器人滚动模式运功中, 机器人质心运动轨 迹如图 33 所示。

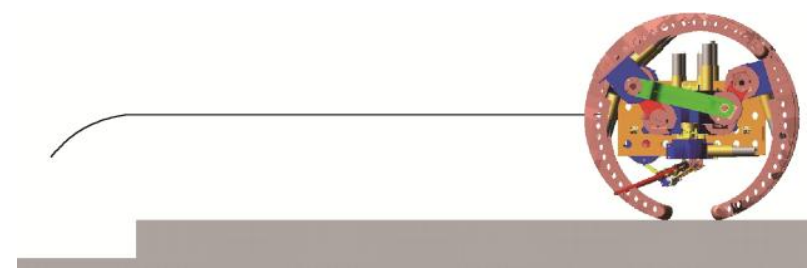

图 33 机器人滚动模式越障质心轨迹

图 34 表示机器人滚动模式越障过程机器人与 地的作用力曲线, 经分析得出, 在机器人触地和机 器人即将越过障碍物时接触力变化较大。该仿真验 证机器人能在地面摩擦力作用下越过 $32 \mathrm{~mm}$ 的障碍, 所需辅助腿的推动力达 $145 \mathrm{~N}$ 。

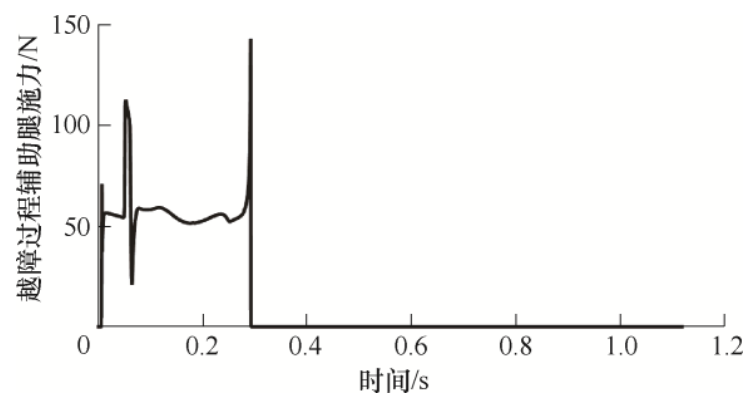

图 34 机器人越障仿真中辅助腿的推动力

滚动模式仿真爬坡和越障结果如表 4 所示。

\begin{tabular}{ccc}
\hline 仿真参数 & 理论值 & 仿真值 \\
\hline 滚动模式爬坡角度 $/\left(^{\circ}\right)$ & $\approx 4.6$ & 5 \\
滚动模式越障高度 $/ \mathrm{mm}$ & $\leqslant 32.06$ & 32 \\
\hline
\end{tabular}

仿真表明机器人能以最大爬坡速度 $1.55 \mathrm{~m} / \mathrm{s}$ 攀 爬 $5^{\circ}$ 斜坡, 越过 $32 \mathrm{~mm}$ 的凸台障碍, 具备一定的爬 坡性能和越障性能。

\section{4 样机试验}

为测试爬-滚机器人的实际性能, 本文研制了爬滚机器人样机并进行试验, 试验样机质量约为 $15 \mathrm{~kg}$, 包络尺寸长宽高为 $480 \mathrm{~mm} \times 1000 \mathrm{~mm} \times 360 \mathrm{~mm}$, 滚 动模式中 “轮” 的直径 $450 \mathrm{~mm}$, 分别在平地、斜坡、 凸台障碍物环境中进行测试，下面根据测试数据讨 论爬-滚机器人的各项性能。

\section{1 爬行模式试验}

\section{1 .1 直行试验}

根据爬行直行步态的运动分析和仿真, 为减小 机器人爬行过程中试验与理论分析值的误差, 应增 大与足端与地面之间的静摩擦。将机器人放置于粗 粘地面后, 通过上位机程序初始化后, 机器人处于 六条腿支撑状态。将直行步态仿真的角度参数值作 为机器人关节的驱动变量输入到关节中, 得到图 35 所示机器人爬行模式直行步态运动过程。

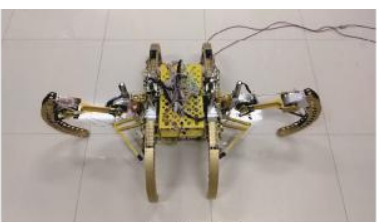

(a) 初始状态

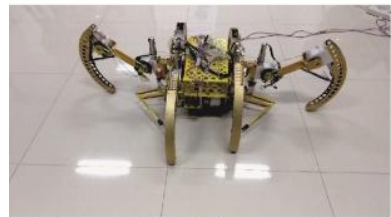

(c) $1 、 4 、 5$ 腿支撑相，2、3、6 摆 动相

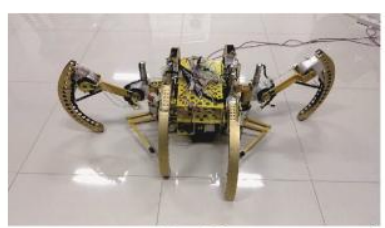

(b) $1 、 4 、 5$ 腿摆动相，2、3、6 支 撑相

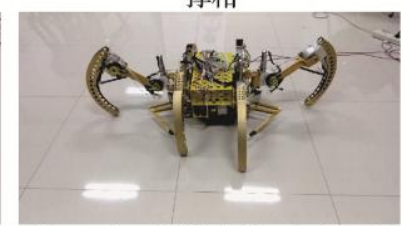

(d) 1、4、5 腿摆动相，2、3、6 支 撑相
图 35 机器人三角步态前行

经测量, 机器人以三角步态前进过程中一个周 期的前进距离为 $190 \mathrm{~mm}$, 侧方向偏移 $7 \mathrm{~mm}$, 直线 度 $93 \%$ 。

\subsection{2 原地转向试验}

将原地转向步态仿真的角度参数值作为样机试 验的关节角度参数, 得到图 36 所示的机器人原地转 向运动步态。为了测量实际转向角度, 将中腿足端 点连成直线, 测量机器人中腿足端点两连线在机器 人原地转向前后的夹角。 


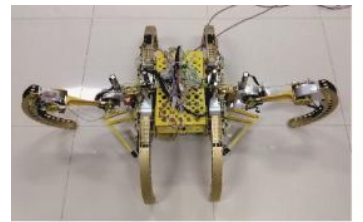

(a) 初始状态

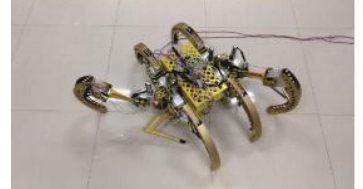

(c) $1 、 2 、 5 、 6$ 腿支撑躯体，3、 4 腿回位

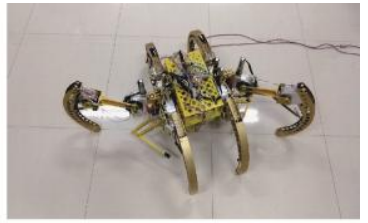

(b) 3、4 腿支撑起躯体转向

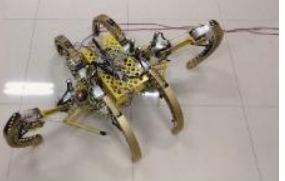

(d) 原地转向结束
图 36 机器人原地转向

测量原地转向前后, 机器人中腿足端连线的夹 角约为 $27^{\circ}$ 。机器人爬行模式试验结果对比如表 5 所示。

表 5 爬行模式主要运动参数对比

\begin{tabular}{lccc}
\hline \multicolumn{1}{c}{ 运动参数 } & 理论值 & 仿真值 & 试验测量值 \\
\hline 爬行一个周期前进距离 $/ \mathrm{mm}$ & 200 & 192 & 190 \\
爬行一个周期的侧移量 $/ \mathrm{mm}$ & 0 & 2 & 7 \\
转向模式转向角度 $\left(^{\circ}\right)$ & 30 & 26.67 & 27 \\
\hline
\end{tabular}

从表 5 中可以得出样机试验值与仿真值近似相 等, 和理论计算值相差较小, 误差产生原因主要是 前行和转向过程中地面对机器人运动产生了阻碍, 导致摩擦滑移。此外, 机器人足端是半球形结构, 理论值是按足端尖点进行计算, 腿的长度与理论值 计算值存在差距。

\section{2 滚动模式试验}

\subsection{1 平坦地面滚动试验}

在滚动试验中, 为了提高辅助腿的驱动能力, 辅助腿的足端做防滑处理, 地面尽可能粗粘, 平地 试验过程如图 37 所示, 机器人可以顺利地完成平地 滚动, 图示为一个滚动周期。
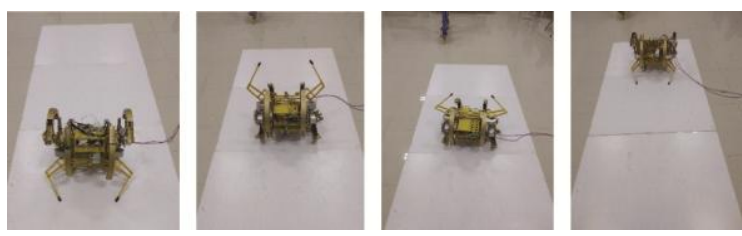

图 37 机器人在平地滚动

\subsection{2 滚动模式爬坡试验}

通过调整斜坡角度, 观察机器人能否滚动一个 周期, 测量出能滚动的最大角度。图 38 为机器人在 斜面上的爬坡运动情况, 经多次试验, 测得机器人 能越过的斜坡大约为 $5^{\circ}$ 。
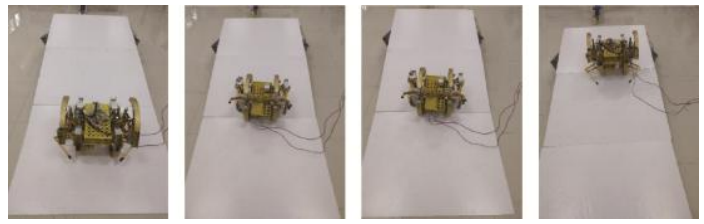

图 38 机器人在斜坡滚动试验

\subsection{3 滚动模式越障试验}

在水平路面上用拼接砖块的方式模拟一个凸台, 不断调整凸台高度, 测得机器人能越过由木板搭建 的障碍物高度为 $30 \mathrm{~mm}$, 越障试验过程如图 39 所示。
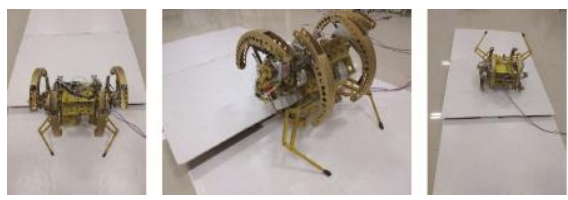

图 39 机器人越障试验

表 6 为机器人试验样机在滚动模式下关键运动 参数对比。

表 6 滚动模式运动特性分析对比

\begin{tabular}{lccc}
\hline \multicolumn{1}{c}{ 分析参数 } & 理论值 & 仿真值 & 试验值 \\
\hline 爬坡角度 $/\left(^{\circ}\right)$ & $\approx 4.6$ & 5 & 5 \\
越障距离 $/ \mathrm{mm}$ & $\leqslant 32.06$ & 32 & 30 \\
\hline
\end{tabular}

从表 6 可以得出, 机器人理论分析的结果与仿 真和试验值近似, 验证了理论分析的正确性。机器 人具有一定的越障和爬坡性能。

\section{5 结论}

(1) 提出一种由六爬行腿和多连杆辅助腿组合 而成的多模式爬-滚机器人方案, 通过腿的形态变化 实现爬行和滚动两种运动模式。该机器人与传统的 轮腿式机器人相比, 自由度更少, 便于控制。

(2) 建立了爬行模式下的运动学模型, 结合运动 学模型分析了基于足端轨迹规划的直行和原地转向 步态, 得到了直行前进步长、转向角度与关节角之间 的关系。建立了滚动模式下平地、斜坡、凸台障碍环 境下滚动模式的受力模型, 结合力约束条件和尺寸约 束条件分析了机器人滚动模式下的爬坡、越障能力。

(3) 通过仿真和试验相结合的方法, 对爬行模 式直行、原地转向运动步态, 对滚动模式爬坡、越 障分别进行了仿真, 进一步开展了样机试验。仿真 和试验结果与理论分析值吻合度高, 验证了爬行模 式、滚动模式运动学分析的正确性和机器人方案设 计的合理性。 


\section{参 考 文 献}

[1] 邓宗全, 范雪兵, 高海波, 等. 载人月球车移动系统综 述及关键技术分析 [J]. 宇航学报, 2012, 33(6): 675-689. DENG Zongquan, FAN Xuebing, GAO Haibo, et al. Review and key techniques for locomotive system of manned lunar rovers[J]. Journal of Astronautics, 2012, 33(6): 675-689.

[2] AMBROSE R, WILCOX B, REED B, et al. Robotics, tele-robotics and autonomous systems roadmap[R]. NASA, Technology Area 04, 2012.

[3] YAMAUCHI B M. PackBot: A versatile platform for military robotics[J]. Proceedings of SPIE, 2004, 5422: 228-237.

[4] Boston Dynamics, Inc. Spot[EB/OL]. [2020-05-14]. https: //www.bostondynamics.com/spot.

[5] ARM P, ZENKL R, BARTON P, et al. Spacebok: A dynamic legged robot for space exploration[C]// International Conference on Robotics and Automation (ICRA 2019), Montreal, Canada, 2019: 6288-6294.

[6] WILCOX B H. Athlete: An option for mobile lunar landers[C]// IEEE Aerospace Conference, Big City, Montana, USA, 2008: 1-8.

[7] Festo, Inc. BionicWheelBot[EB/OL]. [2020-05-14]. https: //www.festo.com.cn/group/zh/cms/13129.htm.

[8] 徐坤, 郑羿, 丁希仑. 六轮腿式机器人结构设计与运动 模式分析 [J]. 北京航空航天大学学报, 2016, 42(1): 59-71.

XU Kun, ZHENG Yi, DING Xilun. Structure design and motion mode analysis of a six wheel-legged robot[J]. Journal of Beijing University of Aeronautics and Astronautics, 2016, 42(1): 59-71.

[9] DING Xilun, ZHENG Yi, XU Kun. Wheel-legged hexapod robots: A multifunctional mobile manipulating platform[J]. Chinese Journal of Mechanical Engineering, 2017, 30(1): 3-6.

[10] 张程煜, 郭盛, 赵福群. 新型轮腿复合机器人的运动分 析及步态研究 [J]. 机械工程学报, 2019, 55(15): 145-153. ZHANG Chengyu, GUO Sheng, ZHAO Fuqun. Motion analysis and gait research of a new wheel-legged compound robot[J]. Journal of Mechanical Engineering, 2019, 55(15): 145-153.

[11] SEENI A, SCHAFER B, HIRZINGER G. Aerospace technologies advancements: Robot mobility systems for planetary surface exploration - state-of-the-art and future outlook: a literature survey[M]. Croatia: InTech, 2010.

[12] CORKE P. Robotics, Vision and control - Fundamental algorithms in matlab[M]. Berlin: Springer, 2011.

[13] BRUNO S, LORENZO S, LUIGI V, et al. Robots modeling, planning and control[M]. London: Springer, 2010.

[14] 程爱祥. 六足机器人非结构化地形下步态生成与腿部 控制研究[D]. 哈尔宾：哈尔滨工业大学, 2014.

CHENG Aixiang. Research on gait generation and leg controlling of a hexapod robot over unstructured terrians[D]. Harbin: Harbin Institute of Technology, 2014.

[15] LIU Zhen, ZHUANG Hongchao, GAO Haibo, et al. Static force analysis of foot of electrically driven heavy-duty six-legged robot under tripod gait[J]. Chinese Journal of Mechanical Engineering, 2018， 31(4): 58-72.

[16] 李进吉. 六足机器人结构参数优化与控制系统设计 [D]. 哈尔滨：哈尔滨工业大学, 2012.

LI Jinji. Structural parameter optimization and control system design of a hexapod robot[D]. Harbin: Harbin Institute of Technology, 2012.

[17] 张赫. 具有力感知功能的六足机器人及其崎岖地形步 行控制研究[D]. 哈尔滨: 哈尔滨工业大学, 2014.

ZHANG He. Research on force sensing based hexapod robot and control for walking on uneven terrain[D]. Harbin: Harbin Institute of Technology, 2014.

[18] 陈刚, 金波, 陈鹰. 六足步行机器人定半径转弯步态 [J]. 浙江大学学报, 2014, 48(7): 1278-1286.

CHEN Gang, JIN Bo, CHEN Ying. Turning gait with constant radius of six-legged walking robot[J]. Journal of Zhejiang University, 2014，48(7): 1278-1286.

[19] 王冠宇, 丁亮，高海波，等. 增强爬坡能力的六足机器 人分步二次规划足力分配算法及试验验证 $[\mathrm{J}]$. 机械工 程学报, 2019, 55(21): 11-20.

WANG Guanyu, DING Liang, GAO Haibo, et al. Decomposed QP CFDA for hexapod robots to enhance the slope-climbing ability and experimental validation[J]. Journal of Mechanical Engineering, 2019, 55(21): 11-20.

[20] ISSA A D N, JARET B M, PABLO A, et al. Axel and DuAxel rovers for the sustainable exploration of extreme terrains[J]. Journal of Field Robotics, 2012, 29(4): 663-685.

作者简介: 张元勋(通信作者), 男, 1984 年出生, 博士, 副教授, 硕士 研究生导师。主要研究方向为空间机构及空间机器人的设计与分析。 E-mail: yuanxun.zh@cqu.edu.cn 黄泽东, 男, 1993 年出生, 硕士研究生。主要研究方向为机器人结构设 计与控制。 